DOI: 10.2478/v10018-007-0013-5

\title{
SPECTRAL CHARACTERISTIC OF POLAR MOTION IN THE 2005- 2006 AND 1999-2000 WINTERS SEASONS
}

\author{
J. Nastula, B. Kolaczek \\ Space Research Centre of the PAS \\ e-mails: nastula@cbk.waw.pl, kolaczek@cbk.waw.pl
}

\begin{abstract}
The Earth's pole moves on the Earth's surface along the spiral curve known as "polhody". Polhody computed from the IERS C04 pole coordinates were compared with those computed after removing successively oscillations with periods shorter than 150,30,10, 2 days. The comparison of these polhody show that the loops in the winter seasons of 20052006 and 1999-2000 are caused by the oscillations of polar motion with periods shorter than 30 days. These short periodical oscillations of geodetic excitation function of polar motion were correlated with those of the atmospheric and oceanic excitation functions. There are high correlations with coefficients equal to 0.8-0.9 during epochs when loops occur.
\end{abstract}

Keywords: Polar motion loops, short periodic oscillations of polar motin and geophysical excitation.

\section{INTRODUCTION}

Variations of the Earth's pole motion have a wide spectral range with periods from hours to decades. There are two large oscillations of pole motion, the free Euleurian wobble known as the Chandler wobble with period of about 433 days and variable amplitude ranging to 300 milliarseconds (mas), and the annual oscillation forced by seasonal displacements of air and water masses with amplitude around 100 mas. The annual oscillation amplitude is slightly variable, but variations of the amplitude of the Chandler wobble are larger (Figure 1). Variations of amplitude of the Chandler wobble have been studied deeply. The correlation of this variation with variations of atmospheric and oceanic excitation functions were found by Brzeziński et al. (2004) and Gross (2000).

The spectrum of the polar motion with periods shorter than semiannual contains several oscillations with periods around 120,60, 50, 30 days and some shorter ones with variable amplitudes of several mas, which are correlated with such oscillations of atmospheric excitation function (Atmospheric Angular Momentum - AAM) and oceanic excitation function (Oceanic Angular Momentum - OAM), [Kolaczek, 1993, 1995; Kosek et al., 1995; Kolaczek et al., 2000] (Figures 2, 3).The Chandler and annual oscillations interfere about every 6.4 years (Figure 4). In the time of the interference the pole moves slowly along the polhody which is the spiral curve with small radius and shorter period fluctuations with small amplitudes become more evident because they are not masked by the fast pole motion (Fig. 5).

At the turn of years 2005-2006 and 1999-2000, the time of interference of the Chandler and annual oscillations the polhody have similar sizes and the loops and high frequency features are visible on the polhody (Figures 4, 5-7). The present high accuracy of polar motion determination allows detection of such small features of the order of 1-2 mas in two weeks. 

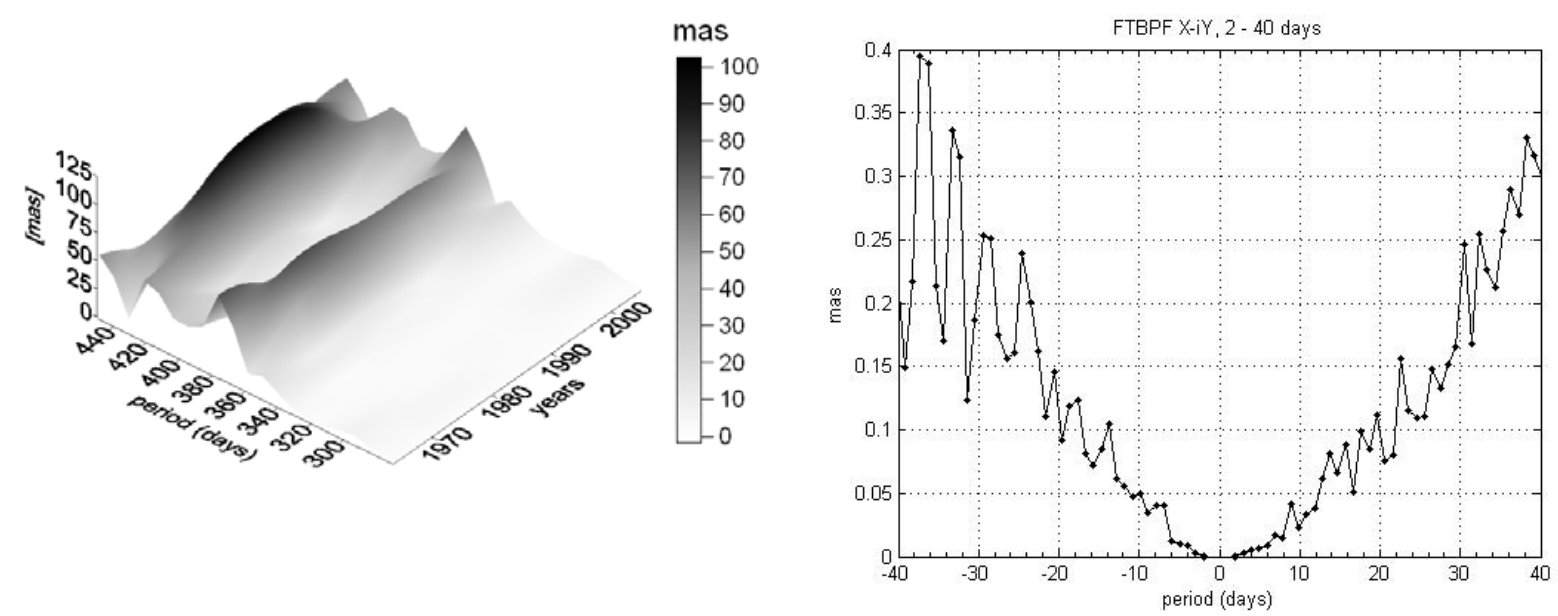

Figure 1. Time variable spectrum of the Figure 2 Spectrum of the polar motion, IERS Chandler and annual oscillations of polar C04; computed by the Fourier Transform motion, IERS C $04 ; \lambda=0.0001$

Band Pass Filter - FTBPF with $\lambda=0.001$ [Kosek, 1995].
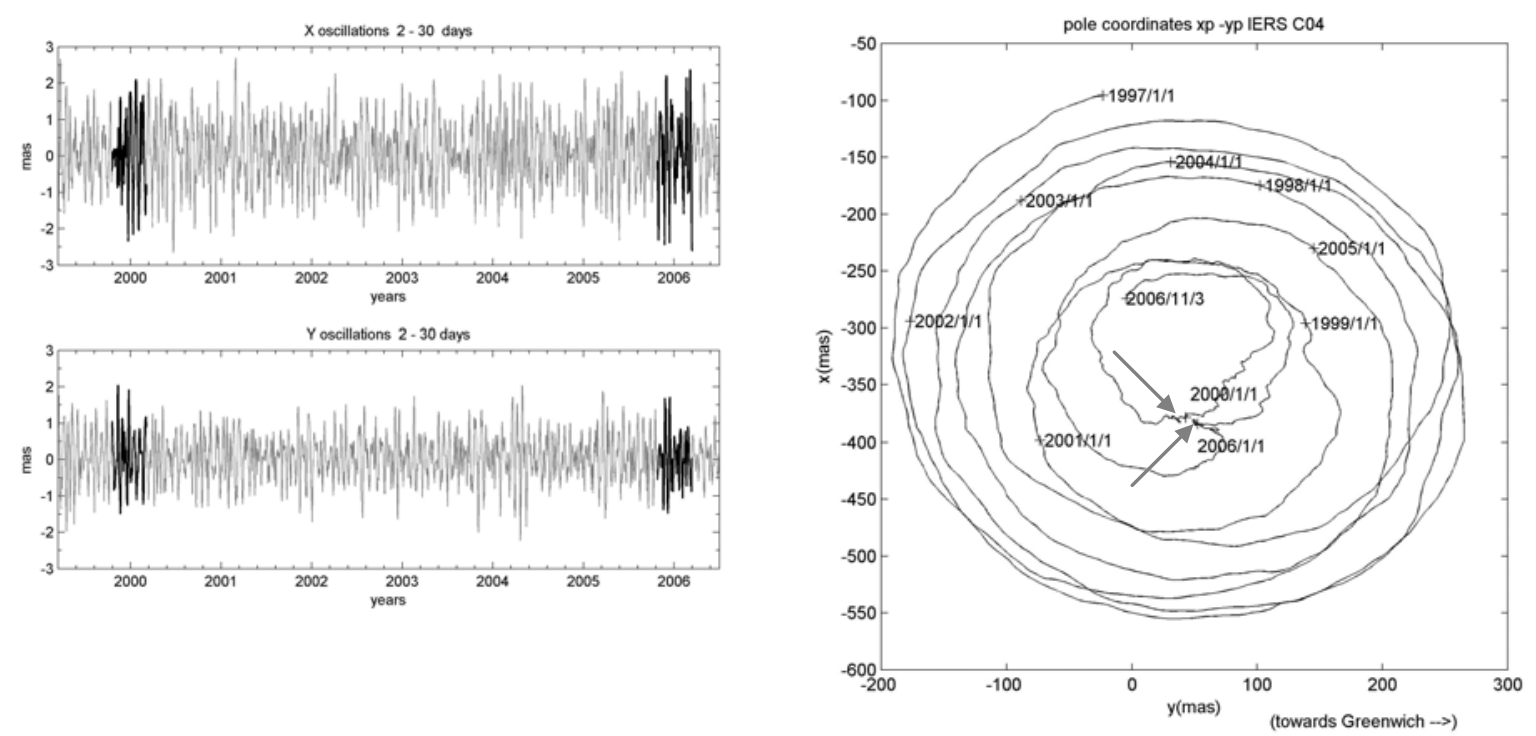

Figure 3. Short periodic oscillations (2-30 Figure 4. Polar motion (IERS C04) for the days) of $x$ and $y$ coordinates of the pole (IERS years 1995-2006, http:/hpiers.obspm.fr C04) filtered by the Butterworth band pass filter.

The loops during the 2005 / 2006 winter season were noticed and analysed by S.B. Lambert et al. (2006). They explained the loops by the combined influence of pressure variations on the continents and oceans. Here we study the spectral characteristic of polar motion in the 20052006 and 1999-2000 winter seasons and correlations of their short period oscillations of geodetic, atmospheric and oceanic excitation functions of polar motion. 

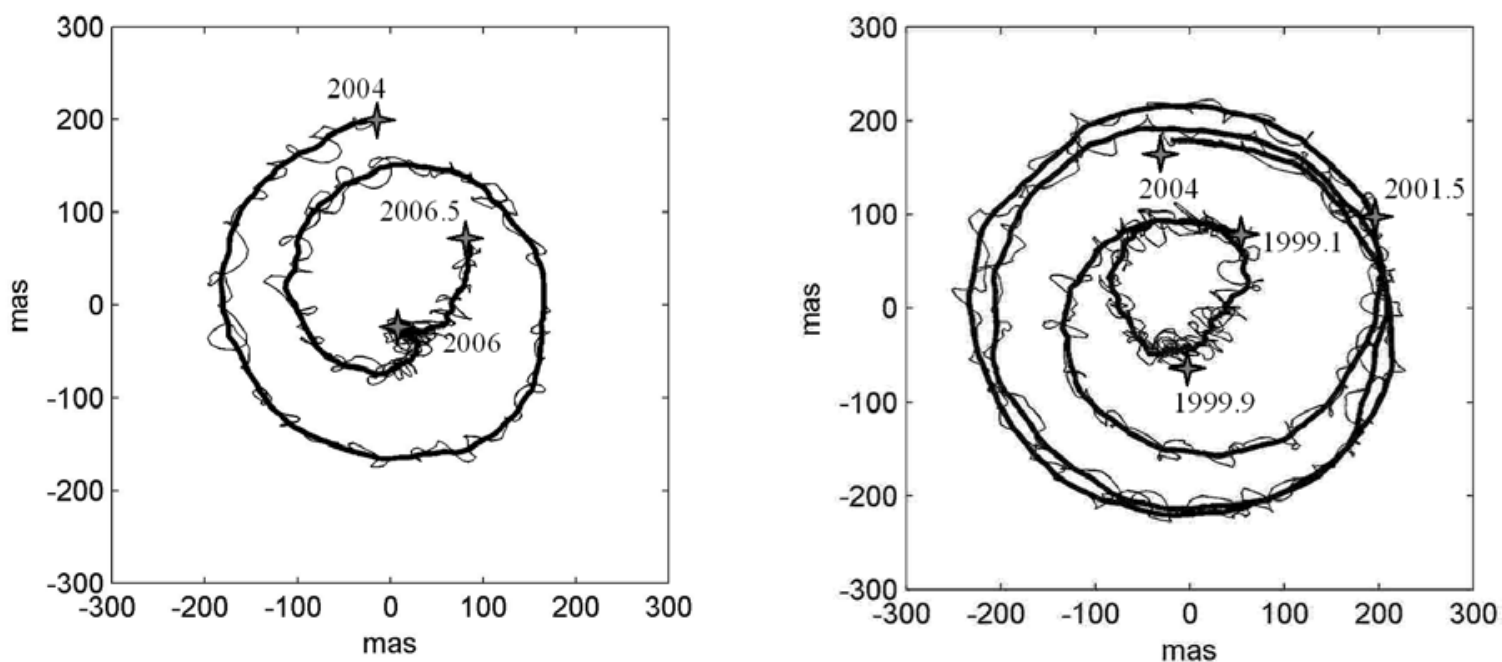

Figure 5. Polar motion computed from the pole coordinates IERS C04 (bold line). Polar motion IERS C04 with the short-period variations of periods less than 30 days increased 10 times (thin line). The maxima of loops are of the order of 2 mas.

\section{ANALYSES}

In order to understand the spectral character of the loops we compare the polhody during the two highlighted time periods computed from the IERS C04 pole coordinates after removing successively short periodical oscillations with periods shorter than $150,30,10,2$ days by the higher-order sine Butterworth band pass filter with eight poles [Otnes et al., 1972] (Figures 6-7). It is seen that the loops and high frequency features are caused mostly by short periodical oscillations with periods shorter than 30 days. To check the influence of atmospheric and oceanic excitation functions on these short disturbances of polar motion the following series were used: AAM (pressure with the IB correction- PIB and wind- W terms) from the Special Bureau for the Atmosphere of the IERS Global Geophysical Fluids Center [Salstein et al., 1993], ECCO-kf049f-6hrchi OAM (bottom pressure- P and currents- C terms) from the Special Bureau for the Oceans [Gross, 2005] of the IERS Global Geophysical Fluids Center and geodetic excitation function computed from the pole coordinates of the IERS C04 by using the Barnes et al. (1983) formula[Barnes et al., 1983; Brzeziński, 1992]. Next all the excitation series were filtered by using the Butterworth band pass filter with the cut of periods of 2 and 30 days [Otnes and Enochson, 1972]. Correlation coefficients between the complex values of equatorial components of these geodetic and atmospheric excitation functions were computed over 30 day intervals starting each 2 days and are shown for the winter seasons of 2005-2006 and 1999-2000. We can notice during the time periods of the loops of the polhody in November-December 2005 and February 2006 as well as NovemberDecember 1999 and January-February 2000, their high correlations with coefficients, of 0.9 and 0.8 respectively (Figure 8). It is necessary to stress that the wind term in AAM increases slightly correlations between atmospheric and geodetic excitation functions of polar motion. Consideration of all the atmospheric and oceanic terms (PIB $+\mathrm{P}+\mathrm{W}+\mathrm{C}$ ) gives the highest correlation (Figure 8). Thus, short period fluctuations are highly correlated with such oscillations of geodetic excitation function. High values of percents of variance explained in geodetic variance by atmospheric $(p i b+w)+$ oceanic $(p+c)$ variances $($ FIG 9) prove that they have enough energy to disturb pole motion. This result is in agreement with the result of Lambert et al. [2006], showing the similarity of atmospheric and ocean bottom pressures with the geodetic excitation of polar motion. 

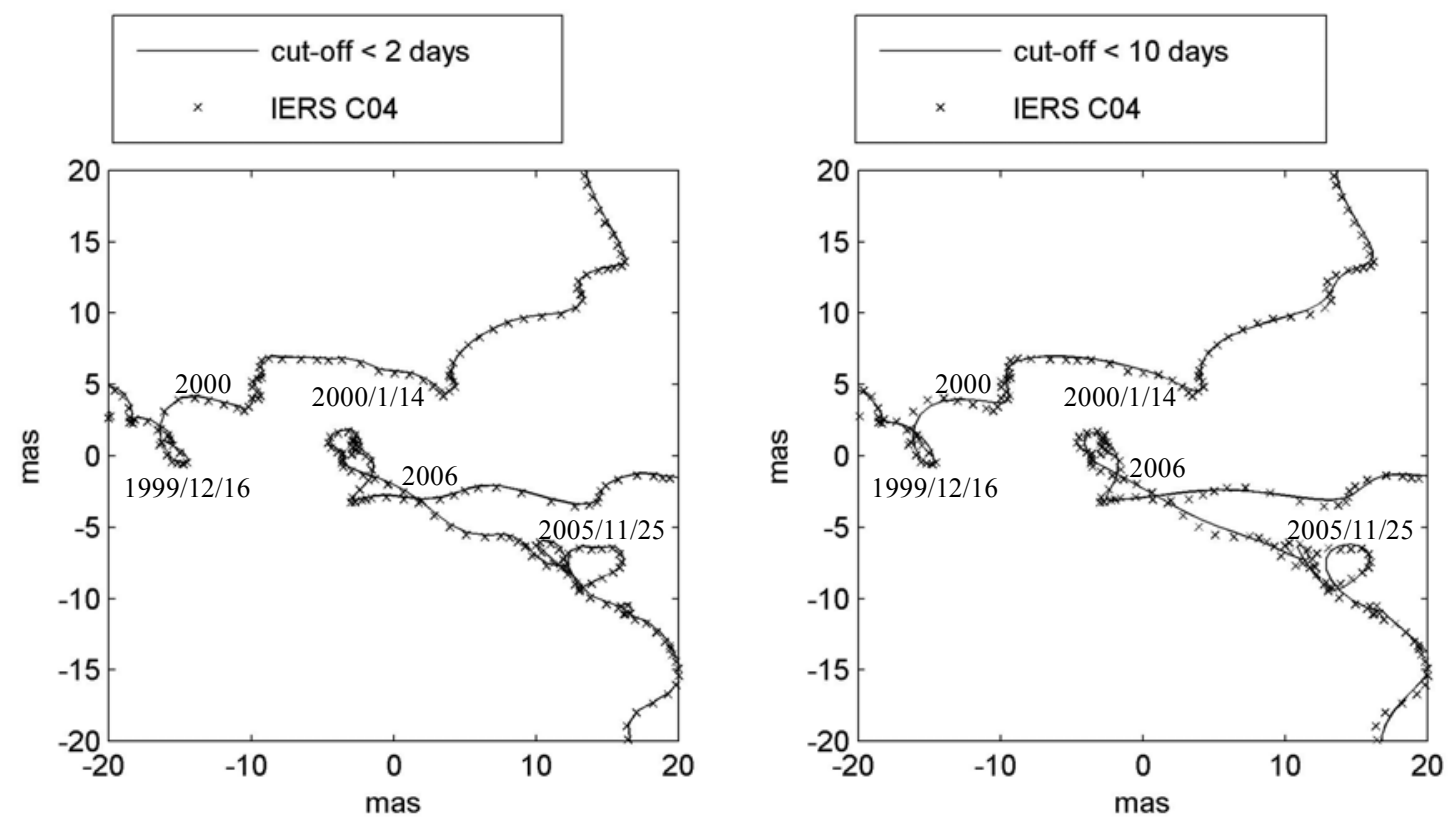

Figure 6. Comparison of polhody - IERS C04, (crosses) with polhody computed from the IERS C04 pole coordinates after removing oscillations shorter than 2 days, (left panel) and shorter than 10 days, (right panel), (solid lines).
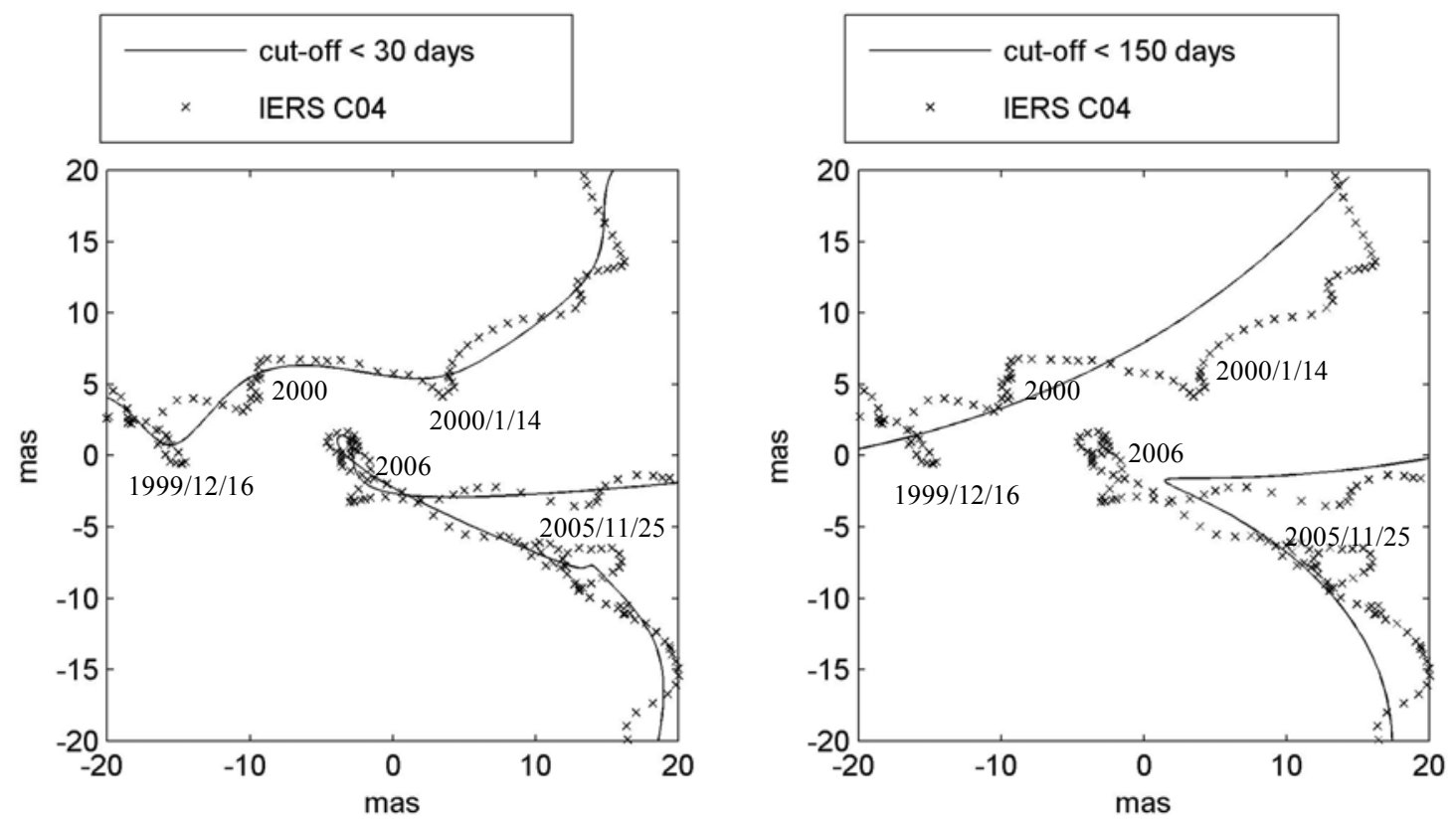

Figure 7. Comparison of polhody - IERS C04, (crosses) with polhody computed from the IERS C04 pole coordinates after removing oscillations shorter than 30 days, (left panel) and shorter than 150 days, (right panel), (solid line).

This paper explains more the spectral character of the loops and the correlations of the considered excitation functions of polar motion (Fig. 8). Consideration of the wind terms and currents terms of atmospheric and oceanic excitation functions respectively increases these correlations such effects were not considered by Lambert et al. (2006). 

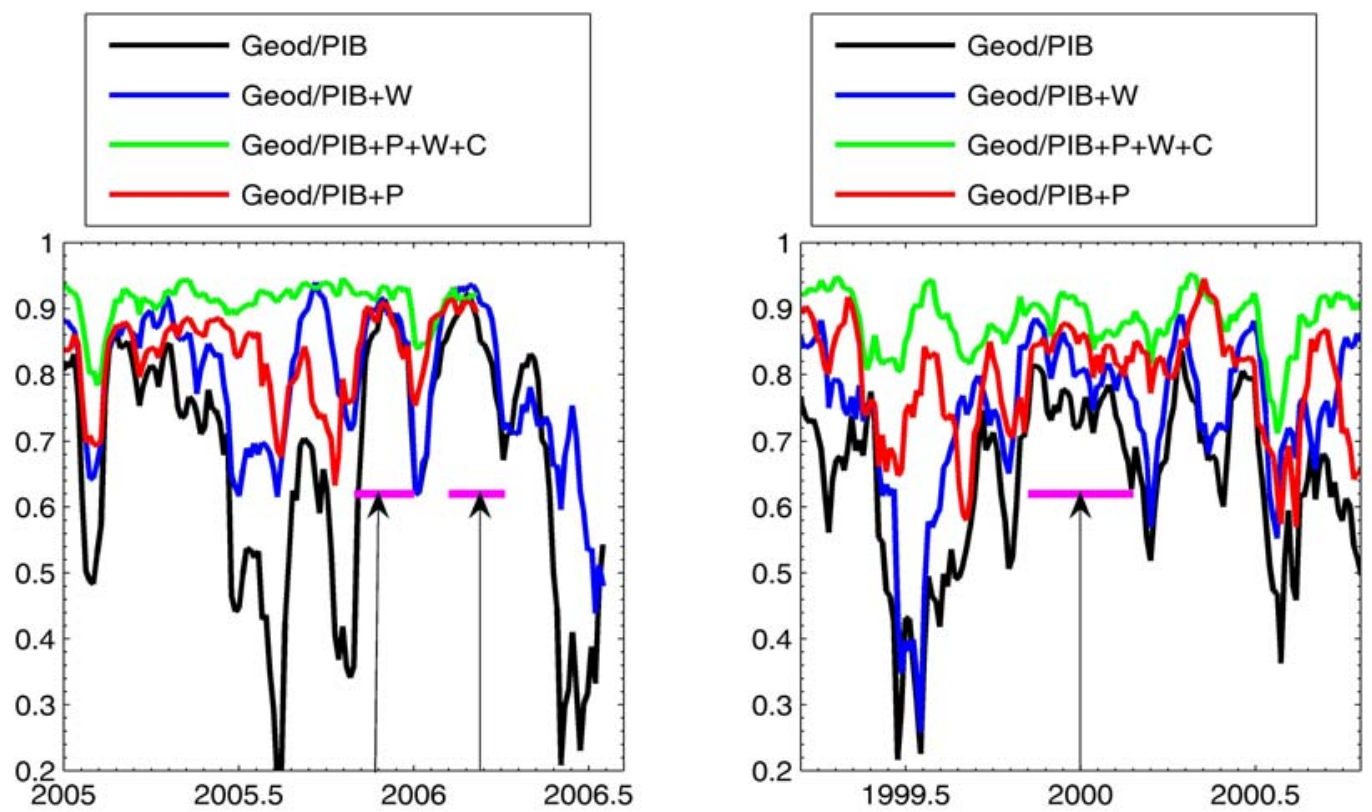

Figure 8. Magnitude of correlation coefficients between the complex values of equatorial components of geodetic, atmospheric and oceanic excitation functions of polar motion filtered by the Butteworth band pass filter, (2-30 days), computed over 30 days interval starting each two days.
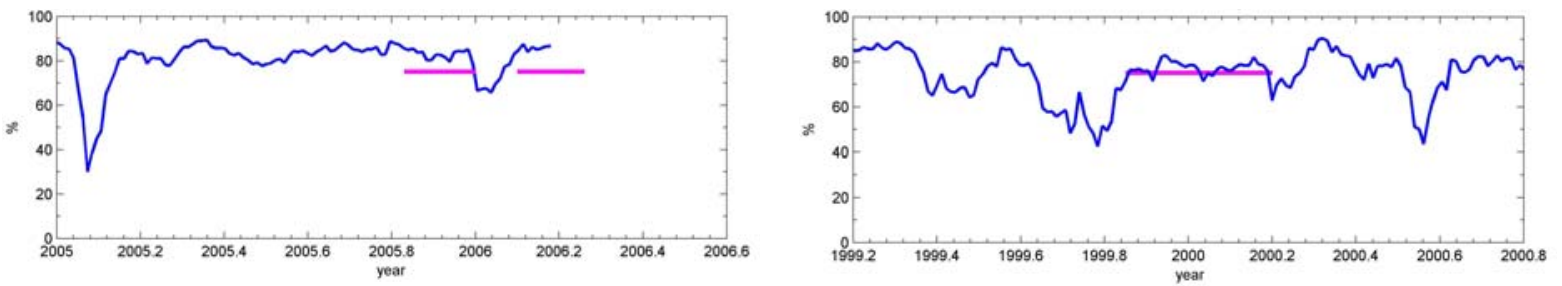

Figure 9. Percents of variances explained in geodetic variances by atmospheric $(\mathrm{pib}+\mathrm{w})+$ oceanic $(p+c)$ variances. Variances denotes modul of variaces of the complex valued series computed over 30 days interval starting each two days.

\section{CONCLUSIONS}

In the periods of destructive interference of annual and Chandler oscillations short oscillations of polar motion with periods shorter than 30 days are responsibly for the newly observed short loops of the polhody in the winter seasons of 2005-2006 and 1999-2000.

There are high correlations between the atmospheric, oceanic and geodetic excitation functions of polar motion in the spectra range from 2 - 30 days in the winter seasons of 20052006 and 1999-2000 at the time when there are loops. The correlation coefficient is of the level of 08-09 in these periods of time. The wind term of the AAM and current term of the OAM respectively increase these correlations between pressure of the AAM, OAM and geodetic excitation functions.

Acknowledgements. The research reported here was supported under the Ministry of Scientific research and Information Technology through project 4T12 E04526. 


\section{REFERENCES}

Barnes R. T. H, R. Hide, A. A. White, and C. A. Wilson (1983) Atmospheric angular momentum fluctuations, length-of-day changes and polar motion, Proc. R. Soc. Lond., A387, 31-73.

Brzeziński A. (1992) Polar motion excitation by variations of the effective angular momentum functions: considerations concerning deconvolution problem, Manuscr. Geodet, 17, 3-20.

Brzeziński A., R.M. Ponte, and A.H. Ali (2004) Nontidal oceanic excitation of nutation and diurnal/semidiurnal polar motion revisited, J. Geophys. Res., 109, B11407, doi:10.10292004JB003054.

Eubanks T.M., J.A. Steppe, J.O. Dickey, R.D. Rosen, and D.A. Salstein (1988) Causes of rapid motions of the Earth's pole, Nature, 334, 115-119.

Gross R.S. (2000) The excitation of the Chandler wobble, Geophys. Res. Lett., 27(15) 23292332.

Gross R. S. Fukumori, I. and Menemenlis, D., (2005) Atmospheric and Oceanic Excitation of Decadal-Scale Earth : Orientation Variations, J. Geophys. Res., vol. 110, B09405.

Kalnay E., et al. (1996) The NMC/NCAR 40-year reanalysis project, Bull. Am. Meteorol. Soc., 77(3), 437-471.

Kołaczek B. (1993) Variations of Short Periodical Oscillations of Earth Rotation. Proc. of the 156th Symp. of the IAU held in Shanghai, China, Sep. 15-19, 1992. Developments in Astrometry and Their Impact on Astrophysics and Geodynamics. I.I Mueller and B. Kolaczek (eds). pp. 291-296. Kluwer Academic Publishers. Dordrecht/Boston/New York.

Kołaczek B. (1995) Short Period Variations of Earth Rotation. Proc. Journees 1995 "Systemes de Reference Spatio-Temporels", Warsaw, Poland, Sep. 18-20, 1995. pp 147-154.

Kołaczek B., W. Kosek, H. Schuh (2000) Short period oscillations of Earth rotation, Proceedings of the IAU Colloquium 178, Polar Motion: Historical and Scientific Problems, pp. 533-544.

Kosek W. (1995) Time Variable Band Pass Filter Spectra of Real and Complex-Valued Polar Motion Series, Artificial Satellites, Planetary Geodesy, 30, Warsaw, Poland, 27-43.

Kosek W., J. Nastula, B. Kolaczek (1995a) Variability of Polar Motion Oscillations with Periods from 20 to 150 Days in 1979-1991. Bulletin Geodesique, Springer Verlag (1995) 69: 308-319.

Lambert S.B., C. Bizouard, and V. Dehant (2006) Rapid variations in polar motion during the 2005-2006 winter season. Geophysical Research Letters, Vol. 33, LXXXXX, doi:10.1029/2006GL026422. 
Otnes R. K., and L. Enochson (1972) Digital Time Series Analysis, John Wiley and Sons, New York.

Salstein D.A., D.M. Kann, A.J. Miller, and R.D. Rosen (1993) The subbureau for atmospheric angular momentum of the International Earth Rotation Service: A meteorological data center with geodetic applications, Bull. Am. Meteorol. Soc., 10, 67-80.

Received: 2007-08-28,

Reviewed: 2007-09-26, by J. Vondrák,

Accepted: 2007-11-08. 